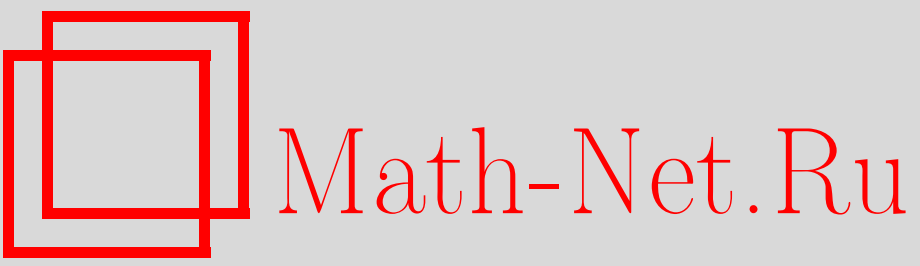

В. В. Вершинин, Инварианты Васильева и косы с особенностями, УМН, 1998, том 53, выпуск 2, 141-142

DOI: https://doi.org/10.4213/rm47

Использование Общероссийского математического портала Math-Net.Ru подразумевает, что вы прочитали и согласны с пользовательским соглашением

http://www.mathnet.ru/rus/agreement

Параметры загрузки:

IP : 54.81 .137 .203

26 апреля 2023 г., 12:21:31 


\title{
ИНВАРИАНТЫ ВАСИЛЬЕВА И КОСЫ С ОСОБЕННОСТЯМИ
}

\author{
В. В. ВЕРшинин
}

Инварианты, определенные В. А. Васильевьм [1], применимы для изучения узлов и зацеплений в трехмерных многообразиях [2]-[4]. Классические теоремы Дж. Александера и А. А. Маркова установили тесные взаимосвязи между теорией зацеплений и теорией кос. В контексте инвариантов Васильева - это связи между зацеплениями с особенностями и косами с особенностями, где под особенностями понимаются только двойные точки трансверсальных самопересечений. Эти связи изучались Дж. Баесом [5] и Дж. Бирман [6].

Целюю настоящей заметки является перенесение резултатов Дж. Баеса [5] на зацепления в телах с ручками. В нашем подходе мы используем группу кос в теле с ручками, которая изучалась А. Б. Сосинским [7] и автором [8], обобщения теорем Дж. Александера и А. А. Маркова для кос с особенностями, доказанные Дж. Бирман [6] и Б. Гемейном [9], а также относительньй вариант теоремы А. А. Маркова, доказанный С. Ламбропулу и К. Рурком [10]. Доказательства Дж. Баеса проходят с необходимыми изменениями для случая тел с ручками.

Пусть $S B_{k}$ есть моноид кос с особенностями из $k$ нитей [5], [6], который назьвается также моноидом Баеса-Бирман или обобщенным моноидом кос. Он имеет образующие $\bar{\sigma}_{i}, \bar{\sigma}_{i}^{-1}, \bar{a}_{i}, i=$ $1, \ldots, k-1$, и соотношения

$$
\begin{aligned}
& \bar{\sigma}_{i} \bar{\sigma}_{j}=\bar{\sigma}_{j} \bar{\sigma}_{i}, \text { если }|i-j|>1, \\
& \bar{a}_{i} \bar{a}_{j}=\bar{a}_{j} \bar{a}_{i}, \text { если }|i-j|>1, \\
& \bar{a}_{i} \bar{\sigma}_{j}=\bar{\sigma}_{j} \bar{a}_{i}, \text { если }|i-j| \neq 1, \\
& \bar{\sigma}_{i} \bar{\sigma}_{i+1} \bar{\sigma}_{i}=\bar{\sigma}_{i+1} \bar{\sigma}_{i} \bar{\sigma}_{i+1}, \\
& \bar{\sigma}_{i} \bar{\sigma}_{i+1} \bar{a}_{i}=\bar{a}_{i+1} \bar{\sigma}_{i} \bar{\sigma}_{i+1}, \\
& \bar{\sigma}_{i+1} \bar{\sigma}_{i} \bar{a}_{i+1}=\bar{a}_{i} \bar{\sigma}_{i+1} \bar{\sigma}_{i}, \\
& \bar{\sigma}_{i} \bar{\sigma}_{i}^{-1}=\bar{\sigma}_{i}^{-1} \bar{\sigma}_{i}=1 .
\end{aligned}
$$

Геометрически $\bar{\sigma}_{i}$ соответствует стандартной образующей группы кос $B r_{k}$, канонически вложенной в $S B_{k}$, а $\bar{a}_{i}$ представляет пересечение $i$-й и $(i+1)$-й нитей.

В качестве аналогичного объекта в теле с ручками рода $g$ естественно рассматривать подмоноид $S B_{n}^{g}$ моноида $S B_{g+n}$, в котором первые $g$ нитей остаются незаплетенными и непересекающимися. Группа кос $B r_{n}^{g}$ в теле с ручками рода $g$ [7], [8] задается образующими $\tau_{k}, k=1,2, \ldots, g$; $\sigma_{i}, i=1, \ldots, n-1$ и соотношениями

$$
\left\{\begin{aligned}
\sigma_{i} \sigma_{j} & =\sigma_{j} \sigma_{i}, \quad \text { если }|i-j|>1, \\
\sigma_{i} \sigma_{i+1} \sigma_{i} & =\sigma_{i+1} \sigma_{i} \sigma_{i+1} \\
\tau_{k} \sigma_{i} & =\sigma_{i} \tau_{k}, \quad \text { если } k \geq 1, i \geq 2, \\
\tau_{k} \sigma_{1} \tau_{k} \sigma_{1} & =\sigma_{1} \tau_{k} \sigma_{1} \tau_{k}, \quad k=1,2, \ldots, g \\
\tau_{k} \sigma_{1}^{-1} \tau_{k+l} \sigma_{1} & =\sigma_{1}^{-1} \tau_{k+l} \sigma_{1} \tau_{k}, \quad k=1,2, \ldots, g-1 ; \quad l=1,2, \ldots, g-k .
\end{aligned}\right.
$$

Эта группа рассматривается как подгруппа классической группы кос $B r_{g}+n$ из $g+n$ нитей, в которой косы из $B r_{n}^{g}$ оставляют первые $g$ нитей незаплетенными. Тогда $\tau_{k}, k=1,2, \ldots, g$, определяются как следующие косы: $\tau_{k}=\bar{\sigma}_{g} \bar{\sigma}_{g-1} \cdots \bar{\sigma}_{k+1} \bar{\sigma}_{k}^{2} \bar{\sigma}_{k+1}^{-1} \cdots \bar{\sigma}_{g-1}^{-1} \bar{\sigma}_{g}^{-1}$. Элементы $\sigma_{i} \in B r_{n}^{g}$ соответствуют элементам $\bar{\sigma}_{i+g} \in B r_{g+n}$.

Пусть $K$ есть коммутативное колцо с единицей, $K[\varepsilon]$ - кольцо многочленов от одной переменной $\varepsilon$ над $K, K S B_{n}^{g}$ - полугрупповая алгебра моноида (полугруппы с единицей) $S B_{n}^{g}$ над $K$.

ОПредЕлЕнИЕ 1. . Алгеброй Васильева $V_{n}^{g}$ назьвается фактор-алгебра алгебры $K S B_{n}^{g} \otimes$ $K[\varepsilon]$ по идеалу, порожденному соотношениями: $\sigma_{i}-\sigma_{i}^{-1}=\varepsilon a_{i}$.

Гомоморфизм вложения $S B_{n}^{g} \rightarrow S B_{g+n}$ индуцирует гомоморфизм алгебр $\alpha: V_{n}^{g} \rightarrow V_{n+g}$ такой, что $\alpha\left(\sigma_{i}\right)=\bar{\sigma}_{g+i}, \alpha\left(a_{i}\right)=\bar{a}_{g+i}, \alpha\left(\tau_{k}\right)=\bar{\sigma}_{g} \bar{\sigma}_{g-1} \cdots \bar{\sigma}_{k+1} \bar{\sigma}_{k}^{2} \bar{\sigma}_{k+1}^{-1} \cdots \bar{\sigma}_{g-1}^{-1} \bar{\sigma}_{g}^{-1}$. Определим гомоморфизм $v^{g}: K B r_{n}^{g} \rightarrow V_{n}^{g}$ по формулам: $v^{g}\left(\sigma_{i}\right)=\sigma_{i}, v^{g}\left(\tau_{i}\right)=\tau_{i}$. Пусть $K(\varepsilon)$ есть алгебра лорановских многочленов от $\varepsilon$ над $K$.

Работа выполнена при финансовой поддержке Российского фонда фундаментальных исследований (гранты 96-01-01631 и 96-01-00062G). 
ПреДЛОЖЕНИЕ. Гомоморфизм $v^{g} \otimes 1: K B r_{n}^{g} \otimes K(\varepsilon) \rightarrow V_{n}^{g} \otimes_{K[\varepsilon]} K(\varepsilon)$ является изоморфизмом.

Пусть $\mathscr{L}$ есть инвариант зацеплений со значениями в $K$, что в нашем случае означает инвариант с точностью до изотопии ориентированных зацеплений в телах с ручками рода $g$. Он каноническим способом продолжается до инварианта зацеплений с особенностями со значениями в $K(\varepsilon)$ по формуле: $\mathscr{L}\left(L_{+}\right)-\mathscr{L}\left(L_{-}\right)=\varepsilon \mathscr{L}\left(L_{\times}\right)$, где $L_{+}, L_{-}$и $L_{\times}$обозначают диаграммы зацеплений c "правым", " левьм" проходом и пересечением в данной точке, оставляющие осталшную диаграмму без изменений. Инвариант $\mathscr{L}$ назьвается инвариантом порядка $d$, если он равен нулю на всех обобщенных зацеплениях, у которых по крайней мере $d+1$ самопересечений. Инвариант порядка $d$ для некоторого $d$ назьвается инвариантом конечного типа.

Для всех $n$ имеются вложения $V_{n}^{g} \subset V_{n+1}^{g}$ и $K B r_{n}^{g} \subset K B r_{n+1}^{g}$. Пусть $V^{g}$ и $K B r^{g}$ есть соответствующие прямые пределы.

ОПРеДЕЛЕниЕ 2. Марковским следомна $V^{g}$ называется $K[\varepsilon]$-линейный гомоморфизм $T: V^{g} \rightarrow$ $E$ в некоторый $K[\varepsilon]$-модуль $E$, удовлетворяющий следующим условиям: $T(x y)=T(y x)$ для всех $x, y \in V^{g}, T\left(x \sigma_{n}^{ \pm 1}\right)=z T(x)$ для некоторого $z \in K$ и для всех $x \in V_{n}^{g} \subset V^{g}$. Аналогично определяется марковский след $\operatorname{tr}: K B r^{g} \rightarrow M$ в некоторый $K$-модуль $M$.

ОПредЕлЕниЕ 3. Марковский след $T: V^{g} \rightarrow K[\varepsilon]$ называется однородным степени $d$, если для каждого $x \in K B r^{g}$ его образ $T(v(x)) \in K[\varepsilon]$ является однородным многочленом от $\varepsilon$ степени $d$.

Для косы $x \in B r_{n}^{g}$ обозначим через $\hat{x}$ ее замыкание по отношению к последним $n$ нитям. Оно рассматривается как зацепление в теле с ручками рода $g$. Для зацепления $L$ обозначим через $L \cup \circ$ незацепленное объединение с незаузленной окружностью, которая также незаплетена с циклами тела с ручками.

ТеОрема. Имеется взаимнооднозначное соответствие между инвариантами зацеплений $\mathscr{L}$ степени d со значениями в $K$ такими, что $z \mathscr{L}(L \cup$ о $)=\mathscr{L}(L)$ для некоторого $z \in K^{*}$ (группь единич кольца $K$ ), и марковским следами $T: V^{g} \rightarrow K[\varepsilon]$, однородными степени d. Инвариант $\mathscr{L}$ определяет след $T$, и обратно, по формуле

$$
T\left(v^{g}(x)\right)=\varepsilon^{d} z^{n-1} \mathscr{L}(\hat{x}) \quad \text { для } x \in B r_{n}^{g} .
$$

Пусть $T_{S}: \mathbf{Z} B r \rightarrow \mathbf{Z}(\varepsilon)$ есть след, построенньй В. Г. Тураевым [11]. Это марковский след с $z=1: T_{S}\left(x \sigma_{n}^{ \pm 1}\right)=T_{S}(x)$ для всех $x \in B r_{n} \subset B r$. Для $i=0, \ldots, g$ определим след $T_{S, i}: \mathbf{Z} B r^{g} \rightarrow \mathbf{Z}(\varepsilon)$ как композицию $\mathbf{Z} B r^{g} \stackrel{\phi}{\longrightarrow} \mathbf{Z} B r^{g-i} \rightarrow \mathbf{Z} B r \stackrel{T_{S}}{\longrightarrow} \mathbf{Z}(\varepsilon)$, где первое отображение определяется по формулам: $\phi\left(\tau_{j}\right)=e$, для $j=1, \ldots, i ; \phi\left(\tau_{j}\right)=\tau_{j-i}$ для $j=i+1, \ldots, g$; $\phi\left(\sigma_{k}\right)=\sigma_{k}$ для всех $k$; а второе есть каноническое вложение. Применим стандартную подстановку $q=\exp (\varepsilon)$ и получим марковский след $T_{i}: V^{g} \rightarrow \mathbf{Z}[[\varepsilon]]$, такой что $T_{i} v^{g}=T_{S, i}$. Имеем $T_{i}=\sum_{d=0}^{\infty} T_{i, d}$, где каждое отображение $T_{i, d}: V^{g} \rightarrow \mathbf{Z}[\varepsilon]$ есть однородньй марковский след степени $d: T_{i, d}\left(x \sigma_{n}^{ \pm 1}\right)=T_{i, d}(x)$ для всех $x \in V_{n}^{g} \subset V^{g}$. Каждый след $T_{i, d}$ дает инвариант зацеплений $\mathscr{L}_{i, d}$ степени $d$ такой, что $\mathscr{L}_{i, d}(\hat{x})=\varepsilon^{-d} T_{i, d}\left(v^{g}(x)\right)$ для $x \in B r r_{n}^{g}$.

\section{СПИСОК ЛИТЕРАТУРЫ}

[1] Vassiliev V. A. Complements of Discriminants of Dmooth Daps: Topology and Applications. Providence RI: Amer. Math. Soc., 1992. [2] Xiao-Song Lin // Topology 1994.. V. 33. №1. P. 45-71. [3] Vassiliev V. A. On Invariants and Cohomology of Spaces of Knots in Arbitrary Manifolds // Preprint: Independent Moscow Univ., 1997. [4] Goryunov V. V. Vassiliev Invariants of Knots in a Solid Torus // Preprint: Univ. of Liverpool, 1997. [5] Baez J. C. // Letters in Math. Physics 1992. V. 26. P. 43-51. [6] Birman J. S. // Bull. of AMS 1993. V. 28. № 2. P. 253-387. [7] Sossinsky A. B. Preparation Theorems for Isotopy Invariants of Links in 3-manifolds // Quantum Groups, Proc. (LNM, No 1510): Berlin a. o., 1992. [8] Vershinin V. V. Homology of Braid Groups in Handlebodies // Preprint No 96/06-2: Univ. de Nantes, 1996. [9] Gemein B. Singular Braids and Markov's Theorem // Preprint: Univ. of Düsseldorf, 1996. [10] Lambropoulou S., Rourke C. // Topology and Its Appl. 1997. V. 78. [11] Turaev V. G. // Invent. Math. 1988. V. 92. P. 527-553. 